\title{
Impact of Mitral Valve Repair in Patients with End-Stage Congestive Heart Failure
}

\author{
John D. Nelson, Pierre Mikhael, Michael A. Wait, Bonnie J. Kuykendall, Kendall A. Nettle, \\ Michael E. Jessen, Dan M. Meyer* \\ Department of Cardiovascular and Thoracic Surgery, University of Texas Southwestern Medical Center, Dallas, USA \\ Email: *Danm.meyer@utsouthwestern.edu
}

Received August 22, 2012; revised September 20, 2012; accepted September 28, 2012

\begin{abstract}
Objective: The utility of mitral valve repair in patients with Mitral Regurgitation (MR) and advanced CHF remains controversial. Methods: 37 patients with MR and Left Ventricular Ejection Fraction (LVEF) $\leq 35 \%$ operated upon between April 2000 and July 2008 were included in the study. Cardiac outcome parameters such as LVEF, left ventricular internal diameter in diastole (LVIDd), NYHA class and mitral regurgitation before and after surgery were retrospectively reviewed. Differences in survival between patients with pre-op EF $\leq 25 \%$ versus pre-op EF $>25 \%$ as well as patients with LVIDd $<6 \mathrm{~cm}$ versus LVIDd $\geq 6 \mathrm{~cm}$ were compared. Significant independent prognostic factors for overall survival were also identified. Results: Operative mortality was $0 \%$ for the group. There were significant reductions in NYHA Class $(p=0.0004)$, mitral regurgitation $(p<0.0001)$ and LVIDd $(p=0.021)$ after surgery. There was significant increase in LVEF after surgery $(p=0.010)$. There were no significant differences in cardiac outcome changes between patients with pre-op EF $\leq 25 \%$ versus pre-op EF $>25 \%$. There were no significant differences in cardiac outcome changes between patients with LVIDd $<6 \mathrm{~cm}$ versus LVIDd $\geq 6 \mathrm{~cm}$. Moreover, there were no significant differences in overall survival between patients with pre-op EF $\leq 25 \%$ versus pre-op EF $>25 \%$, and between patients with LVIDd $<6 \mathrm{~cm}$ versus LVIDd $\geq 6 \mathrm{~cm}$. There were no significant independent prognostic factors for mortality. Conclusions: MV repair in patients with low LVEF and MR can be performed safely, with significant improvement in LVEF and symptom profile. No survival difference were noted between those patients with severely depressed LVEF or those with elevated ventricular dimensions (LVIDd) when compared to those with less severe but still significant cardiac impairment. Consideration should be given to these patients as an option prior to transplantation.
\end{abstract}

Keywords: Mitral Valve Repair; Mitral Regurgitation; Heart Failure

\section{Introduction}

End-stage congestive heart failure patients with progresssive left ventricular systolic dysfunction may develop significant Mitral Regurgitation (MR). This is typically due to left ventricular dilation and geometrical remodeling, displacement of the papillary muscles, ultimately leading to improper valvular coaptation. Multiple studies have shown that MR associated with congestive heart failure is associated with increased mortality [1]. However, without question, the utility of mitral repair in patients with mitral regurgitation and advanced congestive heart failure remains controversial. In addition to medical management and cardiac transplantation, a group of these patients have undergone Mitral Valve repair (MVr) or Replacement (MVR) as another option. Both these procedures have been shown to have favorable structural and symptomatic outcomes [2,3]. However, despite these

"Corresponding author. early reports showing potential benefit of surgery in these patients, more recent studies have been less supportive; showing no clear survival benefit provided by MVr over medical therapy for patients with severe left ventricular dysfunction [4]. Long-term survival advantage has not been clearly identified in subsequent studies [5]. Moreover, adjuvant therapies to mitral valve repair or replacement to aid reverse remodeling are not currently available [6,7].

The aim of this study was to identify a subgroup of patients with MR left ventricular dysfunction that may benefit from mitral valve repair and MVR procedures. In a retrospective review, predictors of morbidity and mortality were identified by correlating pre-operative, postoperative, recent follow-up echocardiogram parameters, NYHA class functions, and surgical outcomes in patients suffering from severe left ventricular dysfunction with preoperative ejection fractions less than or equal to $35 \%$. Outcomes such as quality of life and survival which may 
be impacted and may parallel those results seen in other therapies were discussed.

\section{Methods}

Upon obtaining Institutional Review Board approval for this study, data from the Society of Thoracic Surgeons Outcomes database for patients who had undergone mitral valve surgery was reviewed. Patients undergoing MV repair or replacement at University of Texas Southwestern affiliated hospitals between January 2000 and December 2010 were included in the study. Study patients had symptoms of severe left ventricular dysfunction as diagnosed by an ejection fraction (EF) of less than $35 \%$ preoperatively. Patients undergoing concomitant cardiac procedures were not excluded from the study. Cardiovascular outcomes were compared before and after surgery.

\subsection{Echocardiographic Measurements and Calculations}

All patients in the study underwent echocardiograms preoperatively, post-operatively and at a later follow-up date to determine heart dimensions, ejection fraction and degree of mitral regurgitation. The values measured and recorded were the ventricular parameters, Left Ventricular Internal Dimensions-diastole (LVIDd), left ventricular internal dimension-systole (LVISs), as well as Ejection Fraction (EF), and degree of Mitral Regurgitation (MR), left atrial dimension, and Fractional Shortening (FS) calculated as $[\mathrm{FS}=(\mathrm{EDD}-\mathrm{ESD}) / \mathrm{EDD} \times 100 \%]$.

\subsection{Statistical Methods}

Wilcoxon sign tests or paired t-tests were conducted to investigate if there were significant changes in cardiac outcomes such as LVIDd, EF, NYHA class and mitral regurgitation before and after surgery, with a $\mathrm{p}$ value $\leq$ 0.05 being considered significant. Wilcoxon rank-sum tests and two-sample t-tests were conducted to compare changes in cardiac LVEF and LVIDd when different subsets of patients were compared. Kaplan-Meier survival curves were constructed to estimate the time to death for all patients, as well as when different subset of patients were compared. The differences in overall survival were tested using log-rank tests. Stepwise Cox regression analysis was conducted to identify significant independent prognostic factors for overall survival.

\section{Results}

\subsection{Patient Population}

37 patients were included in the study, 20 males and 17 females. The mean age was 60.7 years, with a range of 36 to 79 years. The baseline characteristics included the frequency of hypertension $(73 \%)$, coronary artery disease $(59 \%)$, angina $(27 \%)$, congestive heart failure $(100 \%)$, and the baseline grade of MR $3-4^{+}(89 \%)$. Mean followup was 27 months.

\subsection{Surgical Procedures}

There were $31(83.9 \%)$ mitral valve repair surgeries and six (16.1\%) mitral valve replacements. All MVr surgeries included placement of an annuloplasty ring, five Cosgrove Rings and 26 Carpentier Edwards Physio Rings. Nineteen of the MVr surgeries were annuloplasties only and nine of the MVr procedures included valve reconstruction. All six MVR patients received chordal-sparing mechanical valve replacements. Thirteen patients also received one or more Coronary Artery Bypass Grafts (CABG) at the time of surgery.

\subsection{Cardiac Parameters}

There were significant reductions in NYHA class $(\mathrm{p}=$ $0.0004)$, mitral regurgitation $(\mathrm{p}<0.0001)$, and LVIDd ( $\mathrm{p}$ $=0.021$ ) after surgery (Table 1). There was significant increase in EF after surgery $(p=0.010)$. Kaplan-Meier survival curves comparing survival in patients with different cardiac profiles are shown in Figure 1. There were no significant differences in cardiac outcome changes between patients with pre-op $\mathrm{EF} \leq 25 \%$ versus pre-op EF $>25 \%$, and between patients with LVIDd $<6 \mathrm{~cm}$ versus LVIDd $\geq 6 \mathrm{~cm}$. Looking at a more severely affected left ventricles, even in patients with an $\mathrm{EF}<20 \%$, there was no difference in survival between these groups within the cohort. However, there was a trend towards poorer survival

Table 1. Changes of cardiac parameters before and after surgery in heart failure patients.

\begin{tabular}{|c|c|c|c|c|}
\hline \multirow{2}{*}{ Variable } & Pre-Operation & Post-Operation & Difference & \multirow{2}{*}{ p-value ${ }^{1}$} \\
\hline & Mean (SD)/Median (Range) & Mean (SD)/Median (Range) & (95\% CI)/Median (Range) & \\
\hline LVIDd cm & $5.9 \pm 0.73$ & $5.48 \pm 1.00$ & $-0.4846(-0.8151$ to -0.1542$)$ & 0.0057 \\
\hline $\mathrm{EF}$ & $0.28 \pm 0.07$ & $0.36 \pm 0.15$ & $0.0748(0.0249$ to 0.1247$)$ & 0.0047 \\
\hline NYHA Class & $3(1-4)$ & $2(1-4)$ & $-1(-2$ to 1$)$ & $0.0003^{*}$ \\
\hline
\end{tabular}

$1, *$ represent p-values from Wilcoxon sign test, the rest of the p-values are from paired t-test. 


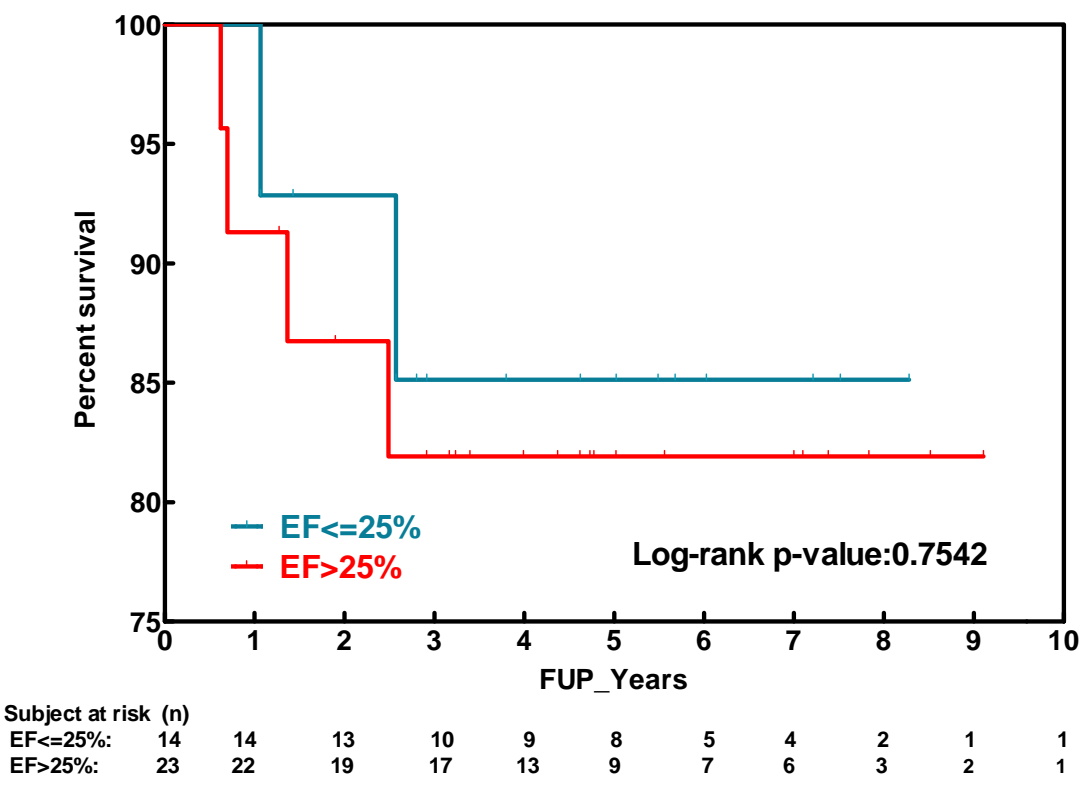

Figure 1. Kaplan-Meier survival curves for all patients, patients with pre-op EF $\leq 25 \%$ versus pre-op EF $>25 \%$.

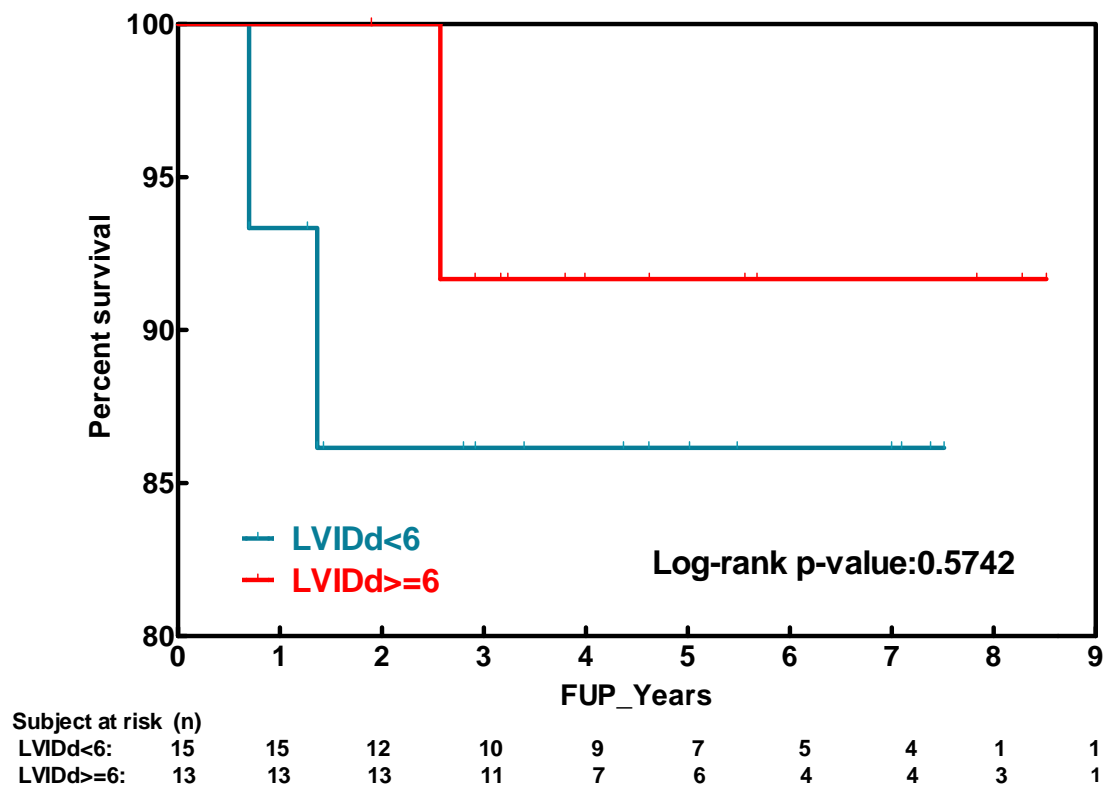

Figure 2. Kaplan-Meier survival curves for all patients, with LVIDd $<6 \mathrm{~cm}$ versus LVIDd $\geq 6 \mathrm{~cm}$.

in patients with an EF less than $20 \%$. Also, in more dilated ventricles, those with a LVIDd greater than $6.5 \mathrm{~cm}$, there was no evidence of a worse survival than patients with LVIDd less than $6.5 \mathrm{~cm}$. However, there was a trend towards lower survival for those patients with LVIDd greater than $6.5 \mathrm{~cm}$. There were no operative mortalities in this series of 37 patients. Stepwise Cox regression analysis shows that there were no significant independent prognostic factors for mortality.

\section{Discussion}

Mitral valve repair in patients with end-stage non-ischemic dilated cardiomyopathy had intuitive appeal and acceptable surgical outcomes when first introduced as an option for this patient population. Studies by Bolling and colleagues [2], and more recently Rukosujew et al. [8] reported excellent results in this group of patients known to have few options other than orthotopic heart transplant. From both an anatomic and physiologic perspective, the reduction of regurgitation by improving leaftlet coaptation has offered intermediate term symptomatic relief in many studies including the current report. The reduction of the degree of mitral regurgitation equated to a significant decrease in LVIDd and significant improvement of NYHA class congestive heart failure. Murakami and colleagues 
[9], in a series with a greater population of patients with ischemic cardiomyopathy than the current report, demonstrated excellent five-year survival, paralleling that of orthotopic heart transplantation. In the multivariate analysis, they did find a group of patients with LVEF $<25 \%$ having a lower short and long-term survival. Their results may help to further identify the subgroup of patients with low LVEF that may benefit from mitral valve repair.

However, while there may be some realignment of the papillary muscles with reverse remodeling of the left ventricle, it is clear that the myocardial dysfunction that occurs with MR in this situation is a left ventricular problem. Perhaps this is the limitation of repair in this group of patients, and why the contemporary results with chordal sparing MV replacement do not differ significantly from those with MV repair [8]. Indeed, attention to the ventricular dilatation and the ability to halt the remodeling that does occur, is key to the long-term impact of this intervention. The importance of preoperative LV dimensions in offering surgery to these patients may influence the potential for reverse remodeling. Many authors have used a LVIDd greater than $6.5 \mathrm{~cm}$ as the upper limit of ventricular dilatation that could demonstrate reverse remodeling after mitral valve repair or replacement. Our study found no survival difference in patients with LVIDd less than or greater than $6.0 \mathrm{~cm}$. However, although no statistical significance was noted in the cohort with LVIDd greater than $6.5 \mathrm{~cm}$, there was a trend towards a worse survival in this more dilated group. This finding again suggests the extent of left ventricular dilatation may be the limiting factor regarding the utility of reparative approaches to these patients.

Efforts to address the left ventricle have actually had positive results although no studies to date have had the definitive results to change clinical practice. In a report by Acker and associates [7], they performed a subgroup analysis of patients undergoing mitral valve repair and replacement as part of the Acorn clinical trial, randomizing patients to the use of the CorCap ${ }^{\circledR}$ cardiac support device. Multiple indices of reverse remodeling were noted in this trial including significant reductions in left ventricular end diastolic volumes and sphericity index. A more recent publication by these investigators [10] demonstrated sustained improvement in left ventricular structure and function after mitral valve surgery for up to 5 years. Their report supported the use of the Acorn CorCap ${ }^{\circledR}$ device at the time of mitral valve repair for patients with nonischemic congestive heart failure with severe left ventricular dysfunction and symptomatic with significant mitral regurgitation.

Another recent attempt to address the ventricular contribution to the outcomes in these patients was reported by Grossi et al. [11]. Using another device designed to reshape the ventricular anatomy that affects both the mitral valve annulus and papillary muscle orientation in patients with dilated cardiomyopathy, subgroup analysis was performed using the Coapsys ${ }^{\circledR}$ device. As part of the RESTOR-MV trial (Randomized Evaluation of a Surgical Treatment for Off-Pump Repair of the Mitral Valve), they showed that the treatment group (CABG plus Coapsys ${ }^{\circledR}$ device) $(\mathrm{n}=74)$ had a significantly greater decrease in LVIDd compared to the patients undergoing CABG plus mitral valve repair $(n=75)$. More importantly, the Coapsys ${ }^{\circledR}$ group showed a survival advantage over the control group at 2 years. Complication-free survival was also greater in the treatment group. Future utilization of interventions such as this may allow for more durable impact with MV repair in these patients as well as offering surgery to patients with even more profoundly affected ventricles.

A true quantification of the affected ventricle was addressed in a report from Ciarka and colleagues [12] who used sophisticated echocardiographic measurements to predict which patients with advanced congestive heart failure and severe mitral regurgitation would have benefit from mitral valve repair. They found that patients with recurrent MR had had increased preoperative posterior and anterior leaflet angles, tenting height, tenting area, and LV sphericity index compared to the patients without recurrent MR. Of the different parameters of mitral and LV geometry, the distal mitral anterior leaflet angle and posterior leaflet angle) were independent determinants of $\mathrm{MR}$ at mid-term $(2.6 \pm 1.6$ years $)$ follow-up. Interesting, they concluded that the etiology of the heart failure may not be the important factor but rather the state of the impaired ventricle as reflected by their echocardiographic measurements. The degree of distal mitral leaflet tethering and posterior mitral leaflet tethering predicted the durability of mitral valve repair in patients with congestive heart failure and mitral regurgitation.

Multiple studies including the current report have demonstrated acceptable long-term survival after mitral valve repair in this severely affected patient population. Overall Kaplan-Meier 5-year survival in this study exceeded $80 \%$. Others have shown that the severity of MR and enlarged left-ventricular end-systolic dimensions are independently associated with mortality [13]. MVr or replacement does offer a likely survival advantage over medical management and until a solution for the ventricular remodeling that may occur can be attained, MV repair may facilitate a delay in orthotopic heart transplant for this group of patients.

\section{Limitations}

This study is limited by both the retrospective nature of the study and the small cohort size. Clearly, there was no control group. No patients that underwent surgical intervention were matched with patients undergoing medical 
therapy. With few exceptions, patients underwent placement of undersized annuloplasty rings, $26 \mathrm{~mm}$ for females, $28 \mathrm{~mm}$ for males. In patients with severely retracted subvalvar and valvar structures, MV replacement was performed. However, studies referenced in this manuscript have shown that in this patient population, no survival difference between MV repair and chordal-sparing MV replacement can be detected. Comparison between MV repair and OHT is clearly speculative. However, there is precedence for this in the Left Ventricular Assist Device (LVAD) population, as younger patients referred for heart transplantation are being considered for LVAD in an attempt to delay the proven but limited long-term survival of heart transplant recipients. Perhaps the same strategy will be considered for patients with severe mitral regurgitation and dilated cardiomyopathy.

\section{Conclusion}

In conclusion, mitral valve repair in patients with low ejection fractions can be performed safely, with significant improvement in left ventricular ejection fraction and improvements in symptom profiles. No survival difference was noted between patients with severely depressed left ventricular fraction or elevated LVIDd and those less severely affected. Mitral valve repair may be an option in a subset of patients who would otherwise be considered for heart transplantation.

\section{REFERENCES}

[1] B. H. Trichon, G. M. Felker, L. K. Shaw, et al., "Relation of Frequency and Severity of Mitral Regurgitation to Survival among Patients with Left Ventricular Systolic Dysfunction and Heart Failure," American Journal of Cardiology, Vol. 91, No. 5, 2003, pp. 538-543. doi:10.1016/S0002-9149(02)03301-5

[2] S. F. Bolling, F. D. Pagani, G. M. Deeb, et al., "Intermediate-Term Outcome of Mitral Reconstruction in Cardiomyopathy," Journal of Thoracic and Cardiovascular Surgery, Vol. 115, No. 2, 1998, pp. 381-386. doi:10.1016/S0022-5223(98)70282-X

[3] M. H. Crawford, J. Souchek, C. A. Oprian, et al., "Determinants of Survival and Left Ventricular Performance after Mitral Valve Replacement," Department of Veterans Affairs Cooperative Study on Valvular Heart Disease, Circulation, Journal of American Heart Association, Vol. 81, No. 4, 1990, pp. 1173-1181.
[4] A. H. Wu, K. D. Aaronson, S. F. Bolling, et al., "Impact of Mitral Valve Annuloplasty on Mortality Risk in Patients with Mitral Regurgitation and Left Ventricular Systolic Dysfunction," Journal of the American College of Cardiology, Vol. 45, No. 3, 2005, pp. 381-387. doi:10.1016/i.jacc.2004.09.073

[5] N. G. Talwalkar, N. R. Earle, E. A. Earle, et al., "Mitral Valve Repair in Patients with Low Left Ventricular Ejection Fractions: Early and Late Results," Chest, Vol. 126, No. 3, 2004, pp. 709-715. doi:10.1378/chest.126.3.709

[6] T. Feldman, E. Foster, D. G. Glower, et al., "Percutaneous Repair or Surgery for Mitral Regurgitation," New England Journal of Medicine, Vol. 364, 2011, pp. 13951406. doi:10.1056/NEJMoa1009355

[7] M. A. Acker, S. Bolling, R. Shemin, et al., "Mitral Valve Surgery in Heart Failure: Insights from the Acorn Clinical Trial," Journal of Thoracic and Cardiovascular Surgery, Vol. 132, No. 3, 2006, pp. 568-577. doi:10.1016/j.jtcvs.2006.02.062

[8] A. Rukosujew, S. Klotz, H. Welp, et al., "Surgery of Secondary Mitral Insufficiency in Patients with Impaired Left Ventricular Function," Journal of Cardiothoracic Surgery, Vol. 4, 2009, p. 36. doi:10.1186/1749-8090-4-36

[9] M. Murakami, H. Yamaguchi, Y. Suda, et al., "Mitral Valve Repair for 52 Patients with Severe Left Ventricular Dysfunction," Annals of Thoracic Cardiovascular Surgery, Vol. 15, No. 3, 2009, pp.160-164.

[10] M. A. Acker, M. Jessup, S. Bolling, et al., "Mitral Valve Repair in Heart Failure: Five-Year Follow-up from the Mitral Valve Replacement Stratum of the Acorn Randomized Trial," Journal of Thoracic and Cardiovascular Surgery, Vol. 142, No. 3, 2011, pp. 569-574. doi:10.1016/i.jtcvs.2010.10.051

[11] E. A. Grossi, N. Patel, Y. J. Woo, et al., "Outcomes of the RESTOR-MV Trial (Randomized Evaluation of a Surgical Treatment for Off-Pump Repair of the Mitral Valve)," Journal of American College of Cardiology, Vol. 56, No. 24, 2010, pp. 1984-1993. doi:10.1016/j.jacc.2010.06.051

[12] A. Ciarka, J. Braun, V. Delgado, et al., "Predictors of Mitral Regurgitation Recurrence in Patients with Heart Failure Undergoing Mitral Valve Annuloplasty," American Journal of Cardiology, Vol. 106, No. 3, 2010, pp. 395-401. doi:10.1016/j.amjcard.2010.03.042

[13] G. Bajraktari, M. Emini, X. Shabani, et al., "Predictors of Mortality in Medically Treated Patients with Congestive Heart Failure of Nonrheumatic Etiology and Reduced Systolic Function," European Journal of Internal Medicine, Vol. 20, No. 4, 2009, pp. 362-365. doi:10.1016/j.ejim.2008.09.011 